\title{
Chandra Multi-wavelength Plane Survey
}

\author{
Ping Zhao ${ }^{1}$, Jonathan E. Grindlay ${ }^{1}$, Jaesub Hong ${ }^{1}$, \\ Mathieu Servillat ${ }^{2} \&$ Maureen van den Berg ${ }^{3}$ \\ ${ }^{1}$ Harvard-Smithsonian Center for Astrophysics, Cambridge, MA 02138, USA \\ ${ }^{2}$ CEA Scalay, France; ${ }^{3}$ University of Amsterdam, The Netherlands
}

\begin{abstract}
Chandra Multi-wavelength Plane Survey (ChaMPlane) surveys the X-ray point sources discovered by the Chandra X-ray Observatory in the galactic plane in order to constrain the populations of faint $\left(L_{x} \leqslant 10^{33} \mathrm{erg} / \mathrm{s}\right)$ accretion-powered sources in the Galaxy. This multi-wavelength survey includes data from the Chandra archive, as well as optical and infrared images and spectra. This paper summarizes a few selected highlights.
\end{abstract}

Keywords. Chandra X-ray Observatory, Survey, Galactic plane, Accretion, X-ray binaries, CVs

\section{Introduction}

The ChaMPlane Survey is designed to investigate the nature of the serendipitous X-ray point sources discovered by the Chandra X-ray Observatory, with its unprecedented spatial resolution, in the galactic plane $\left(|b| \leqslant 12^{\circ}\right.$ ) (Grindlay (2003), Zhao (2003), Grindlay (2005), Hong (2005), Zhao (2005)). Its science goals are: 1) determine the space density of faint $\left(L_{x} \leqslant 10^{33} \mathrm{erg} / \mathrm{s}\right)$ accretion-powered binaries, mainly accreting white dwarfs in cataclysmic variables (CVs) and neutron stars or black holes in low-mass X-ray binaries in quiescence (qLMXBs) in the Galaxy; 2) measure the Be High-Mass X-ray Binary (BeHMXB) density; and 3) study the population of stellar coronal X-ray sources. The ChaMPlane survey was supported by Chandra Archival and GO proposals, NOAO Long Term Survey programs and the National Science Foundation.

The survey comprises data in X-ray: Chandra archive and our own 840ks Chandra observations on three low extinction windows "south" of the galactic center and the galactic bulge latitude survey; optical photometry: CTIO-4m, KPNO-4m: deep V, R, I, $\mathrm{H} \alpha$ imaging; optical spectroscopy: CTIO-4m, Magellan, MMT, WIYN, FLWO-1.5m; infrared photometry: CTIO-4m, Magellan: J, H, K, Br $\gamma$ imaging. ChaMPlane Fields are selected with the following criteria: 1$)$ galactic latitude $|b| \leqslant 12^{\circ} ; 2$ ) deep ACIS imaging mode ( $\geqslant 20 \mathrm{ksec}$ ); 3) without bright (point or extended) X-ray and optical sources or background. So far we have observed 74 ChaMPlane fields (Mosaic FoV: $36^{\prime \prime} \times 36^{\prime \prime}$ ) which cover about 25 square-degs and 325 ACIS observations (AO1-13) in the galactic plane (see Figure 1). This paper summarizes a few selected highlights of this legacy survey.

\section{Galactic Center}

The Galactic center (SgrA*) was observed 44 times in Chandra Cycle 1-10 with a total exposure time of $1407 \mathrm{ks}$. From a stacked image of 14 of the 44 observations with a total exposure time of $750 \mathrm{ks}$, we found 2857 unique point X-ray sources (Hong (2005b)). We searched for their optical and IR counterparts on the images obtained with the CTIO$4 \mathrm{~m} /$ Mosaic and Magellan-6.5m/PANIC. The results show that over $90 \%$ of the soft (0.5$2.0 \mathrm{keV}$ ) sources, especially those without hard band detection, have candidate optical counterparts. But only about $6 \%$ of the hard $(2.0-8.0 \mathrm{keV})$ sources without soft band detection have candidate optical counterparts. A random coincident test (i.e. shifting the X-ray source coordinates and repeating the matching) shows that, due to the severe 


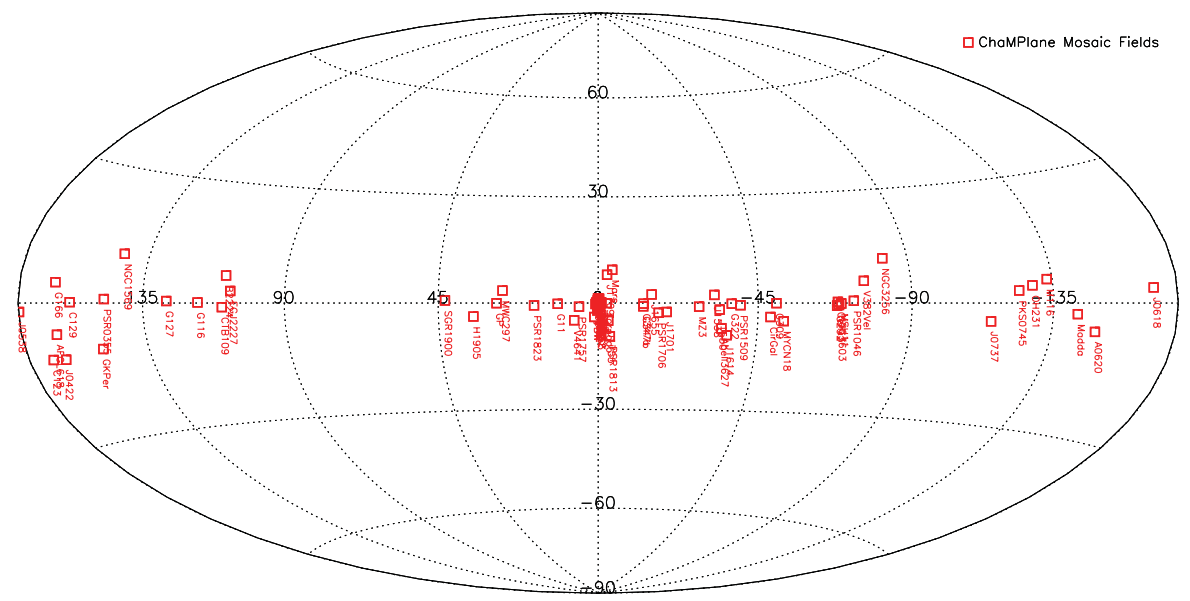

Figure 1. ChaMPlane fields in Galactic coordinates.

extinction towards the Galactic center, most of the optical matches of the hard sources are coincident matches with foreground stars, while most of the matches to the soft sources are their true optical counterparts. Of the 231 soft source counterparts with netBx $(0.3-$ $8.0 \mathrm{keV})>10,179$ have optical spectra taken, 153 of them are classified. Most of them have stellar spectral type F to $\mathrm{M}$. Combining the optical and IR colors and extinction model (Drimmel (2001)), we estimated the distances of all the soft X-ray sources are in the foreground, within $2.2 \mathrm{kpc}$. Figure 2 shows the Chandra sources and their optical counterparts in X-ray quantile color-color diagrams (Hong (2004)).
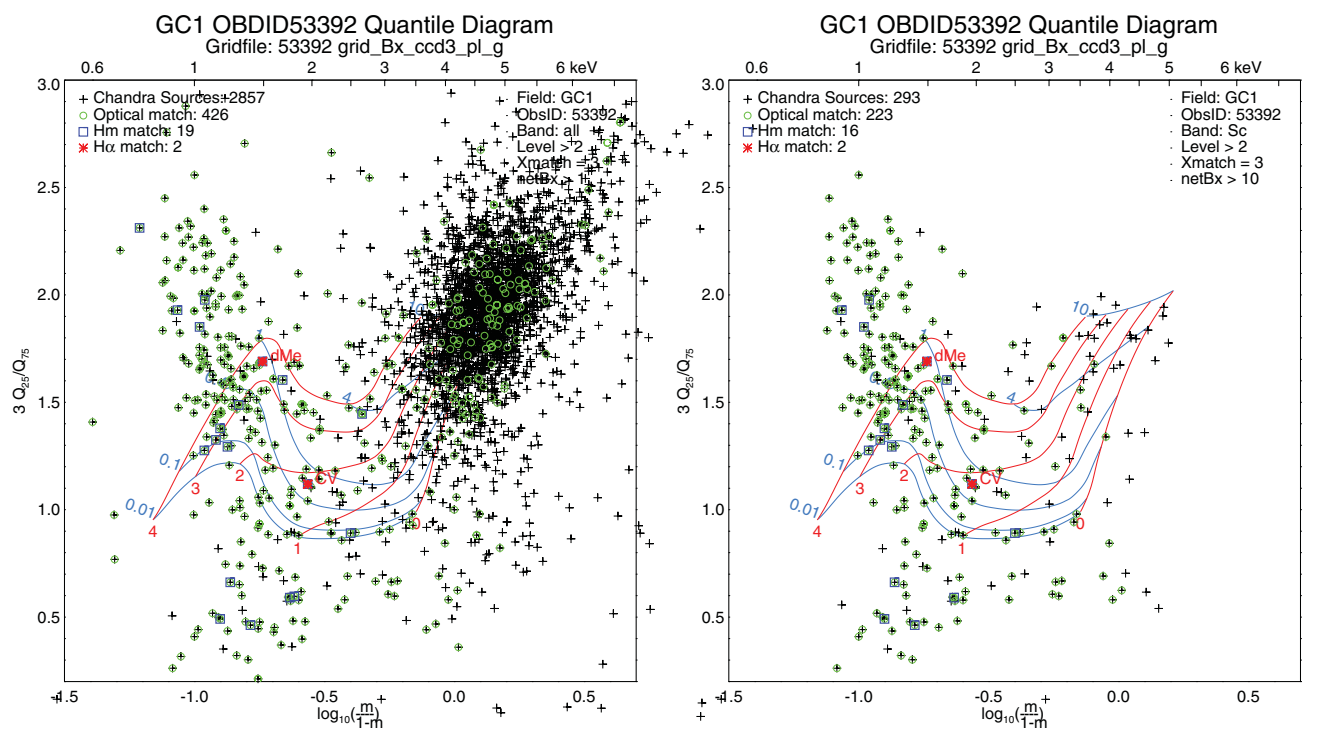

Figure 2. Chandra source quantile diagram (Hong (2004)). Left panel: all sources; Right panel: soft sources. Black crosses are X-ray sources; green circles mark sources with optical matches; red asterisks mark $\mathrm{H} \alpha$ emission sources $(\mathrm{H} \alpha-\mathrm{R} \leqslant-0.3)$; blue boxs mark sources with $\mathrm{H} \alpha-\mathrm{R}$ $\leqslant-0.2$. Soft sources on the left; hard sources on the right. Most of the soft sources have candidate optical matches; most of the hard sources do not have optical matches. There are two Chandra optical matches with $\mathrm{H} \alpha$ emission. The one with the harder X-ray spectrum is a new CV; the one with the softer spectrum is a dMe star (Koenig (2008)). 


\section{Galactic Bulge Latitude Survey}

We conducted the Galactic Bulge Latitude Survey (BLS) in Chandra cycles 7-10, with 36 ACIS-I pointings of $15 \mathrm{ks}$ each. The main goal is to measure the radial gradient of the $L_{x}>10^{31.5} \mathrm{erg} / \mathrm{s}$ source population (primarily CVs and qLMXBs) along latitude vs. longitude in the galactic bulge. The survey is 0.8 degs wide in galactic longitude and extends from \pm 0.2 to \pm 1.6 degs along the galactic latitude, centered at the SgrA*. It covers two $\sim 1.2$ square-degs in the "north" (BLS-N) and "south" (BLS-S) of the galactic plane. The southern tip of the BLS-S is connected with our Chandra observation of the "Limiting Window", a low extinction window $1.4 \mathrm{deg}$ south of the $\mathrm{Sgr}{ }^{*}$ with Av=3.9. We also completed the corresponding optical and IR surveys to cover the same region. Candidate optical counterparts of the X-ray point sources were found from the Mosaic images. Figure 3 shows the combined Chandra color image of our BLS survey with the GCS survey by Wang (2002), and source distributions in different X-ray energy bands.
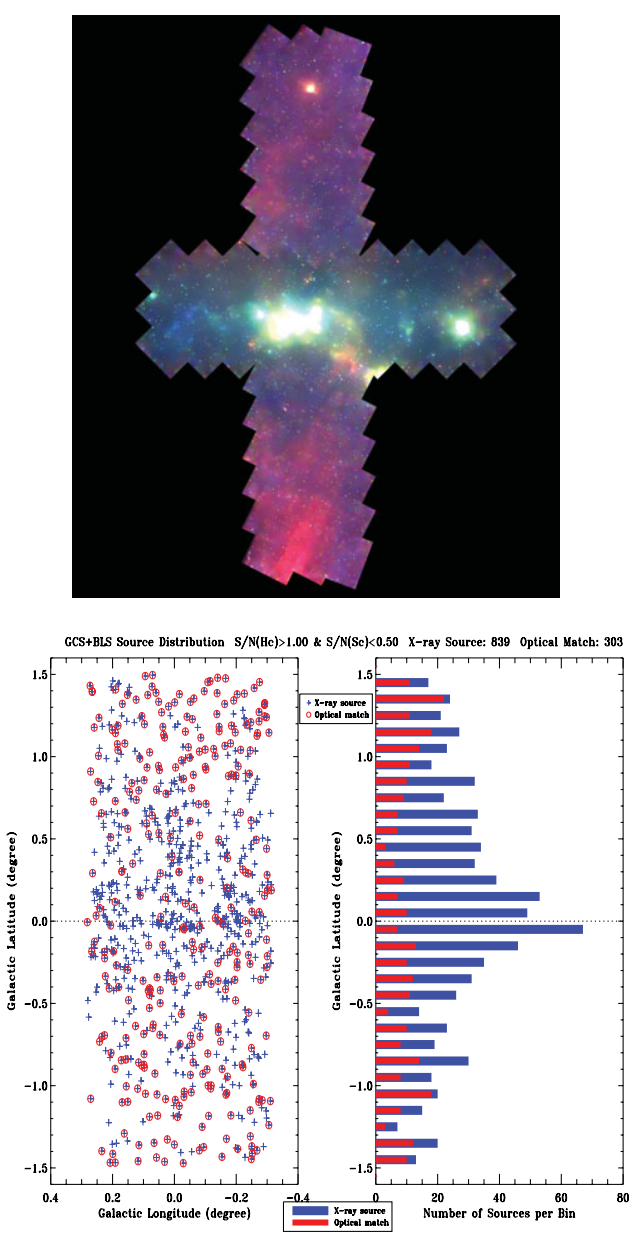
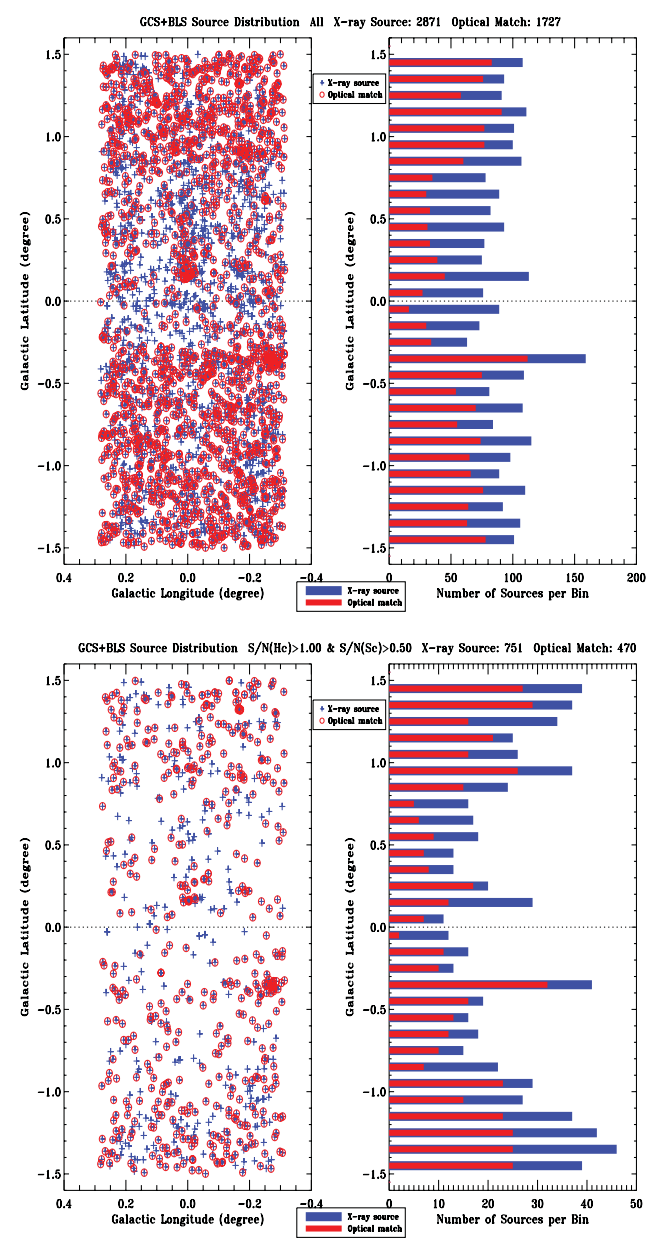

Figure 3. UL: Chandra color image of BLS + GCS survey: red (soft: 1-3keV), green (medium: 3-5 keV), blue (hard: $5-8 \mathrm{keV}$ ). UR: BLS source distribution: All the sources with netBx $\geqslant 1$. LL:Hc $(2.0-8.0 \mathrm{keV})$ band sources without Sc $(0.5-2.0 \mathrm{keV})$ band emission. X-ray source numbers decrease away from the plane, but the percentage with optical matches increases due to decreasing NH. LR: Hc band sources with Sc band emission. X-ray source numbers increase away from the plane, due to decreasing $\mathrm{NH}$, but the percentage with optical matches stays about the same. Most of them are foreground sources. 


\section{Spectral Classification}

We conducted spectroscopic follow-up for identified Chandra candidate optical counterparts and $\mathrm{H} \alpha$ emission sources $(\mathrm{H} \alpha-\mathrm{R} \leqslant-0.3)$ discovered in the optical images, using CTIO-4m/Hydra, Magellan/IMACS and LDSS2, WIYN/Hydra, MMT and FLWO-1.5m, to determine the nature of these sources. So far we have obtained spectra for $6387 \mathrm{ChaM}-$ Plane sources, of which 3999 have been classified. The left panel of Figure 4 shows the distribution of spectral types. The majority of them are stellar coronal sources, especially M stars. Among the 3999 classified sources, we found 25 cataclysmic variables. The right panel shows a sample spectra from one of the CVs found in the galactic center field.
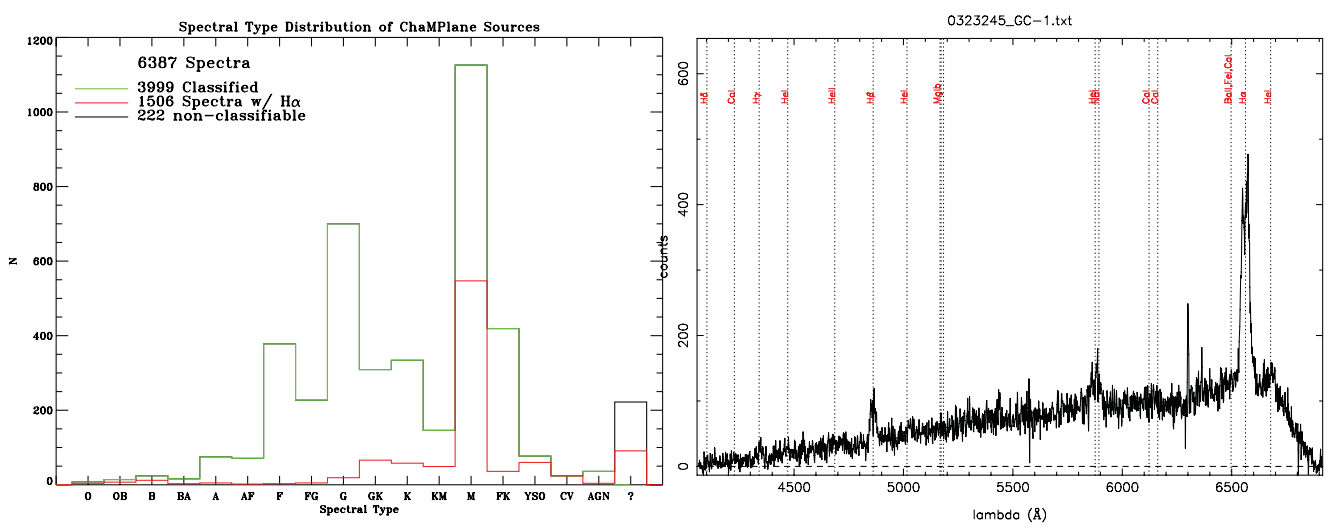

Figure 4. Left: Spectral type distribution of all the classified ChaMPlane sources. Right: Spectrum of a new CV J174421.55-294709.9 discovered in GC5 field.

\section{Summary}

Since year 2000, ChaMPlane has been conducting an unprecedented survey of the Galactic X-ray sources, using the Chandra X-ray Observatory and ground telescopes. So far the survey has produced 74 ChaMPlane fields which cover about 25 square-degs and 325 Chandra (AO1-13) observations. The ChaMPlane database now contains over 15,000 point X-ray sources. Many of them have optical and infrared counterparts. Spectral follow-up were conducted on those optical counterparts. A total of 3999 objects are classified. Among them are 25 new CVs. So far we have published 19 ChaMPlane papers (see our website below). We are building a ChaMPlane legacy database to serve the community, which will provide rich resources for Galactic astronomy in the 21st Century. It is accessible via: ChaMPlane website: http://hea-www.harvard.edu/ChaMPlane/; and NOAO science archive: http://archive.noao.edu/nsa/

\section{References}

Grindlay, J. E. et al. 2003, Astronomische Nachrichten, V324, No.1-2, 57-60

Grindlay, J. E. et al. 2005, ApJ, 635, 920

Drimmel \& Spergel 2001, ApJ, 556, 181

Hong, J. et al. 2004, ApJ, 614, 508

Hong, J. et al. 2005, ApJ, 635, 907

Hong, J. et al. 2009, ApJ, 706, 223

Koenig, X. et al. 2008, ApJ, 685, 463

Wang, D. et al. 2002, Nature, 415, 148

Zhao, P. et al. 2003, Astronomische Nachrichten, V324, No.1-2, 176

Zhao, P. et al. 2005, ApJS, 161, 429 